\title{
Accumulator Pricing
}

\author{
Kin Lam, Philip L.H. Yu, and Ling Xin
}

\begin{abstract}
Accumulator is a highly path dependant derivative structure that has been introduced as a retail financial product in recent years and becomes very popular in some Asian cities with its speculative nature. Despite its popularity, its pricing formula is not well known especially when there is a barrier structure. When the barrier in an accumulator contract is applied continuously, this paper obtains exact analytic pricing formulae for immediate settlement and for delay settlement. For discrete barrier, we also obtain analytic formulae which can approximate the fair price of an accumulator under both settlement methods. Through Monte Carlo simulation, we show that the approximation is highly satisfactory. With price formulae in close forms, this paper further explains how to price the product fairly to fit into its zero-cost structure. The analytic formulae also help in computing the Greeks of an accumulator which are documented in this paper. An asymmetry can be observed here that when the buyer is suffering a loss, risk characteristics like delta and vega are substantially larger than when the buyer is enjoying a profit. This means that losing buyers will be more vulnerable to price changes and volatility changes than winning buyers. This is consistent with another observation in the paper that the value at risk for the buyer can be several times larger than that of the seller.
\end{abstract}

\section{INTRODUCTION}

\section{A. The Accumulator Storm}

$\mathrm{I}^{\mathrm{n}}$ $\mathrm{N}$ the past few months, a structure product called accumulator has aroused a storm of law-suits in Hong Kong and some other Asian cities. On 19th April, 2008 the South China Morning Post in Hong Kong reported: "Once touted as a safe way to tap the bull market, the derivative investment known as the 'accumulator' has become a financial black hole for even sophisticated investors and is causing consternation among regulators. More than 100 Hong Kong investors claim they have lost money - some more than HK\$1 billion - in the unregulated product that allows investors to buy blue-chip stocks at a discount but poses almost unlimited downside risk. That risk, which some investors claim they were never told about, has prompted many to rename the accumulator the 'I kill you later'.....The Securities and Futures Commission (SFC) believes outstanding contracts stand at about US\$23 billion,

Kin Lam is with the Department of Finance and Decision Sciences, The Hong Kong Baptist University, Kowloon Tong, Hong Kong (e-mail: lamkin@hkbu.edu.hk).

Philip L.H. Yu is with the Department of Statistics and Actuarial Science, The University of Hong Kong, Pokfulam Road, Hong Kong (corresponding author, phone: 852-2857-8321; fax: 852-2858-9041; e-mail: plhyu@hku.hk).

Ling Xin is with the Department of Statistics and Actuarial Science, The University of Hong Kong, Pokfulam Road, Hong Kong (e-mail: h0892022@hkusuc.hku.hk). but the market estimates the total reaches US\$40 billion to US\$60 billion, which would affect the stability of the market....." Another law suit was reported by Sydney Morning Herald in the same month : "INVESTMENT banker Greg Bundy is suing stockbroker Goldman Sachs JBWere for misleading and deceptive conduct over an abandoned agreement that he would buy shares at pre-set prices between last September and November this year. ...... Mr. Bundy's statement of claim shows that under the BBM (Buy Below Market) transactions, he would have spent up to \$29.8 million on the three stocks between last September and November this year. The arrangement involved him buying up to $\$ 307,000$ worth of Zinifex shares a week, up to $\$ 192,500$ worth of BHP shares a week and up to $\$ 73,000$ worth of AMP shares a week. ......In fact, between December and February, the share prices of Zinifex, BHP Billiton and AMP fell below the specified purchase prices. In January, Mr. Bundy sold the Zinifex and AMP shares he had bought for a combined loss of $\$ 933,000 \ldots . . . "$

According to Dutch Bank ABN AMRO, the majority of accumulators are bought by Hong Kong investors - who have a high-risk appetite, followed by investors from Singapore. Investors rushed to the products during the market boom but reports of large losses in investing in accumulators have surfaced in the past few months since the share prices started to head south. Despite of its popularity in Asia, investment characteristics of this product are not so well publicized and many investors have difficulty in analyzing the pricing and risk characteristics of this product. This paper attempts to give a rigorous treatment on this product by going through its pricing mechanism and its risk characteristics. In the following, we first provide a brief history of accumulator and then spell out a typical accumulator contract based on which we perform our pricing and risk analysis.

\section{B. A Typical Accumulator Contract}

Accumulator is a highly path dependant derivative structure that has been introduced as a retail financial products in recent years and becomes very popular in some Asian cities with its speculative nature. According to Wystup (2006), accumulator contract linked to foreign currency has been very popular among corporate investment in many countries in European for many years. In 2005, FCStone Trading Company in US started to offer crop linked accumulator contracts for elevators and producers and it could be the earliest accumulator products that can be accessed by retail clients. Soon after, other accumulator contracts become popular in retail market. For example, 
there are FX accumulator issued by Credit Suisse, accumulated boosted forward issued by Calyon and KODA ELI (Knock-out Discounted Accumulative Equity Linked Instrument) issued by Macquarie. In below, we spell out the terms of a typical accumulator contract in which the underlying asset is a particular stock in the equity market.

A typical accumulator contract obligates an investor to buy a quantity of a stock on specified observation days within the term of the contract at a strike price $K$ which is usually set at a discount of the original spot price $S_{0}$ of the stock. $K$ is called the strike price or discounted purchase price in this paper. The observation days are denoted by $t_{1}, t_{2}, \ldots t_{n}$ where $t_{i} \leq N$ and $N$ is the length of the contract term. Note that $K$ remains a constant throughout the life of the contract. Furthermore, if the closing price $S_{i}$ on the $i^{\text {th }}$ observation day is larger than or equal to $K$, the purchase quantity is fixed as $Q$. However, if $S_{i}$ is less than $K$, the purchase quantity is fixed as $2 Q$. The contract also has a knock-out feature in that if the closing price at any time within the contract life exceeds a barrier $H$, the contract will terminate automatically. An accumulator usually has a zerocost structure meaning that there is no premium payment for both parties.

Settlement arrangement is also an important condition in an accumulator contract. It can be immediate settlement or delay settlement. Immediate settlement means that investor will be delivered the fixed quantity ( $Q$ or $2 Q$ ) immediately on day $t_{i}$. In practice, however, immediate settlement is not so common. While the quantity of purchase $(Q$ or $2 Q)$ is fixed on day $t_{i}$, settlement is often done periodically on a weekly or monthly basis. Under a weekly settlement, all stocks to be purchased in the same week will be cleared at the last day in that week. In general, there can be $\mathrm{m}$ settlement days throughout the life of the contract and the settlement days can be different from the observation days. Both immediate and delay settlements will be discussed in this paper. For more precise notation on observation days and settlement days, see Section II.B.

Another detail needs to be noted for an accumulator contract. The knock-out barrier $H$ can be applied discretely or continuously. While quantity fixing is done only at the end of an observation day, a barrier is either applied discretely at the end of all observation days or continuously throughout trading days within the term of the contract. In the former case, we call the barrier a discrete barrier and in the latter case, a continuous barrier. Accumulators with a continuous barrier will be treated in Section II and those with a discrete barrier are treated in Section III.

While it is common for an accumulator to have quantities fixed at $Q$ or $2 Q$, a general accumulator can have a gearing ratio and the purchase quantity can be fixed as $Q$ or $g Q$. For practical purposes, $Q$ is usually set as a multiple of the lot size. In this paper, we choose to discuss an accumulator with $g=2$ and $Q=1$, and refer to this contract as a typical accumulator contract. This paper will analyze the pricing and risk characteristics of this typical contract. If a general contract has a different gearing ratio $g$ and a different quantity $Q$, the formulae provided in this paper can be generalized easily.

The rest of the paper is organized as follows. Section II.A presents the decomposition and replication of accumulator using barrier options. The fair value of a typical accumulator contract with continuous barrier and immediate settlement will then follow from this decomposition. Section II.B deals with the fair value under delay settlement. In Section III, we further provide an approximate pricing formula to cater for discrete barrier. In Section IV, a sample accumulator is analyzed. Section IV.A investigates whether the approximate pricing formula for discrete barrier is accurate or not. Section IV.B provides a way to design an accumulator contract with a zero cost structure. Section IV.C deals with implied volatility and Section IV.D provide the value at risk and the Greeks for the sample accumulator under study. Section V concludes the paper.

\section{PRICING AN ACCUMULATOR WITH A CONTINUOUS BARRIER}

\section{A. Decomposition under Immediate Settlement}

In this subsection, we consider first an accumulator contract with $\mathrm{N}$-day to expiration and with immediate settlement. Under immediate settlement, the pay-off at day $t_{i}(i \leq n)$ is given by

$$
\left\{\begin{array}{cll}
0 & \text { if } \max _{0 \leq \tau \leq t_{i}} S_{\tau} \geq H & \\
S_{t_{i}}-K & \text { if } \max _{0 \leq \tau \leq t_{i}} S_{\tau}<H, \quad S_{t_{i}} \geq K \\
2\left(S_{t_{i}}-K\right) & \text { if } \max _{0 \leq \tau \leq t_{i}} S_{\tau}<H, \quad S_{t_{i}}<K
\end{array}\right.
$$

The pay-off is equivalent to long one up-and-out barrier call option and short two up-and-out barrier put options with expiration time $t_{i}$. Hence, the fair price of an accumulator contract is given by

$$
V=\sum_{i=1}^{n}\left\{C_{\text {ио }}\left(t_{i}, K, H\right)-2 \cdot P_{\text {ио }}\left(t_{i}, K, H\right)\right\}
$$

where $C_{u o}$ represents the fair price of a up-and-out barrier call option and $P_{u o}$ represents the fair price of a up-and-out put option. If we adopt the usual Black-Scholes assumptions of constant risk-free interest rate $(=r)$, constant volatility $(=\sigma)$ as well as a constant payout rate $(=q)$ of the underlying asset, the terms $C_{u о}$ and $P_{u о}$ have closed form solutions. Rubinstein and Reiner (1991) studied binary options with continuously monitored barrier under the Black-Sholes' framework and various kinds of binary barrier options can be priced. Using the results derived by Harrison 
(1985) and by Rubinstein and Reiner (1991), we are able to express $C_{\text {ио }}$ and $P_{\text {ио }}$ as below:

$$
\begin{aligned}
& C_{u о}\left(K, H, t_{i}\right)=S_{0} e^{-q t_{i}}\left\{N\left(x\left(t_{i}\right)\right)-N\left(x_{1}\left(t_{i}\right)\right)+\left(\frac{H}{S}\right)^{2 \lambda}\left[N\left(-y\left(t_{i}\right)\right)-N\left(-y_{1}\left(t_{i}\right)\right)\right]\right\} \\
& -e^{-r t_{i}} K\left\{N\left(x\left(t_{i}\right)-\sigma \sqrt{t_{i}}\right)-N\left(x_{1}\left(t_{i}\right)-\sigma \sqrt{t_{i}}\right)+\left(\frac{H}{S}\right)^{2 \lambda-2}\left[N\left(-y\left(t_{i}\right)+\sigma \sqrt{t_{i}}\right)-N\left(-y_{1}\left(t_{i}\right)+\sigma \sqrt{t_{i}}\right)\right]\right\}
\end{aligned}
$$

$$
\begin{aligned}
& P_{u o}\left(K, H, t_{i}\right)=e^{-r t_{i}} K\left\{N\left(-x\left(t_{i}\right)+\sigma \sqrt{t_{i}}\right)-\left(\frac{H}{S}\right)^{2 \lambda-2} N\left(-y\left(t_{i}\right)+\sigma \sqrt{t_{i}}\right)\right\} \\
& -S_{0} e^{-q t_{i}}\left\{N\left(-x\left(t_{i}\right)\right)-\left(\frac{H}{S}\right)^{2 \lambda} N\left(-y\left(t_{i}\right)\right)\right\}
\end{aligned}
$$

where $N($.$) represents the cumulative standard normal$ distribution function and

$x\left(t_{i}\right)=\frac{\log (S / K)+\left(\mu+\sigma^{2}\right) t_{i}}{\sigma \sqrt{t_{i}}}, x_{1}\left(t_{i}\right)=\frac{\log (S / H)+\left(\mu+\sigma^{2}\right) t_{i}}{\sigma \sqrt{t_{i}}}$,

$\mu=r-q-\sigma^{2} / 2$,

$y\left(t_{i}\right)=\frac{\log \left(H^{2} / S K\right)+\left(\mu+\sigma^{2}\right) t_{i}}{\sigma \sqrt{t_{i}}}, y_{1}\left(t_{i}\right)=\frac{\log (H / S)+\left(\mu+\sigma^{2}\right) t_{i}}{\sigma \sqrt{t_{i}}}$, $\lambda=1+\frac{\mu}{\sigma^{2}}$.

Substitute (3) and (4) into (2), we get the fair value of an accumulator at time 0 as follows:

$$
V=\sum_{i=1}^{n}\left\{\begin{array}{l}
S e^{-q t_{i}}\left[\begin{array}{l}
2-N\left(x\left(t_{i}\right)\right)-N\left(x_{1}\left(t_{i}\right)\right)- \\
\left(\frac{H}{S}\right)^{2 \lambda} N\left(-y\left(t_{i}\right)\right)-\left(\frac{H}{S}\right)^{2 \lambda} N\left(-y_{1}\left(t_{i}\right)\right)
\end{array}\right] \\
-K e^{-r t_{i}}\left[\begin{array}{l}
2-N\left(x\left(t_{i}\right)-\sigma \sqrt{t_{i}}\right)-N\left(x_{1}\left(t_{i}\right)-\sigma \sqrt{t_{i}}\right) \\
-\left(\frac{H}{S}\right)^{2 \lambda-2} N\left(-y\left(t_{i}\right)+\sigma \sqrt{t}_{i}\right) \\
-\left(\frac{H}{S}\right)^{2 \lambda-2} N\left(-y_{1}\left(t_{i}\right)+\sigma \sqrt{t_{i}}\right)
\end{array}\right]
\end{array}\right\}
$$

\section{B. Decomposition under Delay Settlement}

In this subsection, we will price an accumulator with delay settlement. For the sake of easier presentation, we introduce the following notations. Let $T_{i}(i=1,2, \ldots, n)$ denote the settlement day of the quantities fixed at observation day $t_{i}$. While $t_{1}<t_{2}<\ldots<t_{n}$ represent $\mathrm{n}$ different observation days, $T_{1} \leq T_{2} \leq \ldots \leq T_{n}$ may be equal. In fact, there are only $m$ different values in $\left\{T_{i} ; i=1,2, \ldots, n\right\}$ because there are only $m$ different settlement days. This is made possible because even when $t_{i}<t_{j}, T_{i}$ can be equal to $T_{j}$ meaning that both quantities determined on day $t_{i}$ and $t_{j}$ are settled on the same day. Thus the pay-off at observation day $t_{i}$ actually come on the settlement day $T_{i}$. Hence, the pay-off at day $t_{i}$ is the value of a forward contract expiring at day $T_{i}$. With this notation, the pay-off of an accumulator at an observation day $t_{i}$ is given by

$$
\left\{\begin{array}{cll}
0 & \text { if } \max _{0 \leq \tau \leq t_{i}} S_{\tau} \geq H & \\
S_{T_{i}}-K & \text { if } \max _{0 \leq \tau \leq t_{i}} S_{\tau}<H, & S_{t_{i}} \geq K \\
2\left(S_{T_{i}}-K\right) & \text { if } \max _{0 \leq \tau \leq t_{i}} S_{\tau}<H, & S_{t_{i}}<K
\end{array}\right.
$$

The above pay-off is equivalent to long one up-and-out barrier call option on forward contract and short two up-andout barrier put options on forward contract where the options' expiration time is $t_{i}$ and the corresponding forward contract is an agreement to buy the underlying asset at time $T_{i}$ at a price equals to $K$. Hence, the fair price of an accumulator contract with delay settlement is given by

$$
V^{\text {Delay }}=\sum_{i=1}^{n}\left\{C_{u o}^{F}\left(t_{i}, K, H, T_{i}\right)-2 \cdot P_{u o}^{F}\left(t_{i}, K, H, T_{i}\right)\right\}
$$

where $C_{u o}^{F}\left(t_{i}, K, H, T_{i}\right)$ is the price of an up-and-out barrier call option on a forward contract with purchase price equals to $K$ and maturity date equals to $T_{i}$. The option has a barrier $H$ and expires at day $t_{i}$. Using the density functions derived by Rubinstein and Reiner (1991), we can derive the values of the above barrier call and put options on forward contracts. Hence, the value of an accumulator with delay settlement is

$$
V^{\text {Delay }}=\sum_{i=1}^{n}\left\{\begin{array}{l}
S e^{-q T_{i}}\left[\begin{array}{l}
2-N\left(x\left(t_{i}\right)\right)-N\left(x_{1}\left(t_{i}\right)\right) \\
-\left(\frac{H}{S}\right)^{2 \lambda} N\left(-y\left(t_{i}\right)\right)-\left(\frac{H}{S}\right)^{2 \lambda} N\left(-y_{1}\left(t_{i}\right)\right)
\end{array}\right] \\
-K e^{-r T_{i}}\left[\begin{array}{l}
2-N\left(x\left(t_{i}\right)-\sigma \sqrt{t_{i}}\right)-N\left(x_{1}\left(t_{i}\right)-\sigma \sqrt{t_{i}}\right) \\
-\left(\frac{H}{S}\right)^{2 \lambda-2} N\left(-y\left(t_{i}\right)+\sigma \sqrt{t_{i}}\right) \\
-\left(\frac{H}{S}\right)^{2 \lambda-2} N\left(-y_{1}\left(t_{i}\right)+\sigma \sqrt{t_{i}}\right)
\end{array}\right]
\end{array}\right\}
$$

where the functions $x\left(t_{i}\right), x_{1}\left(t_{i}\right)$, etc. are as defined in the last subsection.

\section{PRICING AN ACCUMULATOR WITH A DiscRETE BARRIER}

Under a discrete barrier, an knock-out event happens only if the barrier is breached at market closes of day $t_{i}$. Since pricing formulae (5) and (7) are derived under a continuous barrier setting, they fail to deal with accumulator with a discrete barrier. According to Cheuk and Vorst (1994), using the continuous approach to price a discrete barrier option may lead to remarkable pricing errors. To deal with a discrete barrier, Heynan and Kat (1994) pointed out that the value of a discrete barrier option involves high dimensional 
numerical integration. Boyle and Lau (1994) and Reimer and Sandmann (1995) investigate the application of the binomial model to price the barrier options with discrete barrier. Ritchken (1995) and Cheuk and Vorst (1996) use trinomial trees instead and find out that it has a distinct advantage over that of a binomial tree. However, the methods above are all computationally costly and time consuming.

More recently, Broadie, Glasserman, and Kou (1997) proposed an approximation of discretely monitored barrier option values using continuous formulae with an appropriately shifted barrier. The correction term used to shift the barrier of an up-and-out barrier option is $e^{\beta \sigma \sqrt{T / m}}$ where $\sigma$ is the underlying volatility, $T$ is the tenor of the option, $m$ is the number of barrier monitoring points throughout the tenor and $\beta$ is a constant factor involving the zeta function and is given by $\beta=-\zeta(1 / 2) / \sqrt{2 \pi} \approx 0.5826$. So the corrected barrier is $\widetilde{H}=H e^{\beta \sigma \sqrt{T / m}}$. Under these notations, the value of an upand-out barrier option will be approximated by

$$
V_{\text {Discrete }}(H)=V(\tilde{H})
$$

where $V_{\text {Discrete }}(H)$ represents an approximation of the pricing formula for a discrete barrier $H$ with $m$ barrier monitoring points and $V(\widetilde{H})$ represents the pricing formula for a barrier option with continuous barrier $\widetilde{H}=H e^{\beta \sigma \sqrt{T / m}}$. Because an accumulator can be taken as a combination of options, we can apply this approximation to our pricing formulae. In our case, $T=T_{i}$ for the option component that expires on the $i^{\text {th }}$ trading day, and $m=m_{i}$ is the number of observation days between time 0 to $T_{i}$. Hence $\widetilde{H}_{i}=H e^{\beta \sigma \sqrt{T_{i} / m_{i}}}$ for all $i$. So if we substitute $H$ by $\widetilde{H}_{i}$ in (5), we get the pricing formula for discrete barrier. Similarly, if we substitute $H$ by $\widetilde{H}_{i}$ in (7), we get the pricing formula for delay settlement with a discrete barrier. The numerical Lattice methods developed for discrete barrier options by Broadie, Glasserman, and Kou (1999) showed that this approximation is quite accurate. It works in the case of a single barrier and has been regarded as a very convenient and practical method.

\section{ANALYZING A SAMPLE ACCUMULATOR}

\section{A. How Good is the Approximation to Discrete Barrier Accumulator Pricing}

In this section, we study a numerical example of an accumulator with a discrete barrier, a one year tenor $(T=1)$, daily observation and monthly settlement. We assume there are 21 trading days each month. The underlying security's spot price $S_{0}$ at day 0 is $\$ 100$, the accumulator contract's exercise price $K$ is $\$ 90$ and the discrete barrier $H$ is $\$ 105$, $Q=1$, and gearing ratio $=2$. When the underlying price at the end of a trading day is in the range of 90 to 105 , the accumulation quantity $Q$ is 1 . When price falls below 90 , the effective accumulation quantity equals to 2 . In this example, we assume a constant interest rate of $3 \%$, and assume that the underlying security's dividend payout rate is zero, and volatility is $20 \%$. In the industry, the notional amount of this accumulator is usually calculated by assuming that the underlying price always stays in the range between strike $K$ and barrier $H$ throughout the life time of the contract. This means that the investor has to buy one share at all trading days in the coming year. If $n$ is the total number of trading days in an accumulator contract, the notional amount is $n^{*} Q^{*} K$. In our sample contract, the tenor is one year, $n$ is 252 , and $Q=1$ and the notional amount is $\$ 22,680$. If $Q=1$ represents one lot size of 400 shares, notional amount equals $\$ 9,072,000$.

The objective in this section is to test the accuracy of using (8) as the fair price of the sample accumulator. We follow Broadie, Glasserman and Kou (1997) to compare this approximate value with a value obtained by Monte Carlo simulation. To assess the accuracy of their approach in pricing discrete barrier option, Broadie, Glasserman and Kou (1997) use numerical lattice methods to do comparison. But they also mention that simple Monte Carlo method with 1 million simulations can reach a $95 \%$ confidence interval with error range of 6 percent. Here we compare our analytical result (8) with Monte Carlo simulation result. We improve the simple Monte Carlo method with some variance reduction methods and use 5 million simulations. The numerical results and the analytical results are presented in Table I. The difference is within an acceptable range.

Since the accumulator parameters are designed so that it has a near zero-cost structure, the fair price for the sample accumulator is small. Despite the smallness of its fair value, the percentage difference remains small. The absolute difference $(=\$ 1.885)$ is also of a small magnitude considering the fact that this structure product involves many options with various expiration days.

\section{B. The Fair Value of an Accumulator}

Since an accumulator usually has a zero-cost structure, we can now check whether an accumulator is fairly priced or not by its closed form valuation formula. Using the same example in last Section, the fair price under various volatility and various discount percentage $(=K)$ with fixed $H$

TABLE I

Monte Carlo vs Closed Form VALUe of ACCUMUlator CONTRACT

\begin{tabular}{|c|c|c|c|}
\hline $\begin{array}{c}\text { Monthly } \\
\text { settlement }\end{array}$ & $\begin{array}{l}\text { Closed form } \\
\text { value }\end{array}$ & MC value & $\begin{array}{c}\text { Difference in } \\
\text { percentage }\end{array}$ \\
\hline$V_{\text {discrete }}^{\text {Delay }}$ & -84.845 & -82.96 & $2.27 \%$ \\
\hline
\end{tabular}

$=\overline{105 \text { and } S_{0}=100 \text { can be computed. The results are }}$ tabulated in Table II. Because of its zero-cost structure, the positive values in the upper left corner means that accumulators with these discounted purchase prices $(=K)$ are 
worthy for investors while negative values means those contracts favors the issuers. The exact zero structure purchase prices associated with a given volatility is provided in the last column of Table II.

TABLE II

FAIR VALUES OF ACCUMULATOR CONTRACTS

\begin{tabular}{|c|c|c|c|c|c|}
\hline \multirow{2}{*}{$\begin{array}{l}\text { Volatility } \\
(\sigma)\end{array}$} & \multicolumn{4}{|c|}{ Discounted Purchase Price $K$} & \multirow{2}{*}{$\begin{array}{c}\text { Zero- } \\
\text { structure } \\
\text { discounted } \\
\text { price }\end{array}$} \\
\hline & 78 & 84 & 90 & 96 & \\
\hline $10 \%$ & 2639.5 & 1821.5 & 978.4 & 24.2 & 96.14 \\
\hline $15 \%$ & 1785.8 & 1108.4 & 369.8 & -499.5 & 92.70 \\
\hline $20 \%$ & 1217.4 & 604.0 & -82.2 & -883.4 & 89.32 \\
\hline $25 \%$ & 790.0 & 211.6 & -437.1 & -1180.8 & 86.04 \\
\hline $30 \%$ & 445.2 & -109.3 & -727.2 & -1423.3 & 82.86 \\
\hline $35 \%$ & 155.2 & -380.6 & -972.4 & -1629.2 & 79.80 \\
\hline $40 \%$ & -95.5 & -615.9 & -1185.4 & -1809.6 & 76.84 \\
\hline
\end{tabular}

For example, assume that $S_{0}=100, H=105, n=252, r=$ $0.03, q=0.00, \sigma=20 \%$. For a zero-cost accumulator with monthly settlement to be fairly priced, a fair discounted purchase price is shown to be 89.32 .

\section{Implied Volatility}

Options' implied volatility is the volatility implied by the market price of the options based on a pricing model. In other words, given a particular pricing model, it is the volatility that yields a theoretical option value equal to the market price. Implied volatility is usually a measure that is used to monitor the expectation about the fluctuation of the underlying. Given the closed form valuation formulae (5), (7) and (8), it is now possible to calculate the implied volatility of accumulator contracts. Since accumulator is a zero-cost product, we need to find the volatility that makes the fair price equal to zero. Keeping other conditions of the sample accumulator contract unchanged, we calculate the implied volatilities under various discounted prices and barrier prices. The results are tabulated in Table III. Here strike and barrier values are represented in percentage of original spot price.

TABLE III

IMPLIED VOLATILITIES OF ACCUMULATOR CONTRACTS

\begin{tabular}{cccccc}
\hline \multirow{2}{*}{$\begin{array}{c}\text { Barrier } \\
\text { level }\end{array}$} & \multicolumn{5}{c}{ Discounted Purchase Price $K$} \\
\cline { 2 - 6 } & 80 & 84 & 88 & 92 & 96 \\
\hline 107 & $\mathbf{3 6 . 0 6 \%}$ & $\mathbf{2 9 . 5 5 \%}$ & $23.30 \%$ & $17.26 \%$ & $11.31 \%$ \\
\hline 105 & $\mathbf{3 4 . 6 3 \%}$ & $\mathbf{2 8 . 1 6 \%}$ & $21.97 \%$ & $16.00 \%$ & $10.16 \%$ \\
\hline 103 & $\mathbf{3 3 . 1 0} \%$ & $\mathbf{2 6 . 6 8} \%$ & $20.54 \%$ & $14.63 \%$ & $8.91 \%$ \\
\hline \hline
\end{tabular}

Suppose an investor anticipates a volatility of $25 \%$ in the future one year. This investor will find the barrier-strike combination in the upper left corner (bold area in Table III) favorable because implied volatilities in those cells have implied volatility larger than $25 \%$.

\section{Value at Risk and the Greeks}

In this section, we compute some risk characteristics of the sample accumulator contract with details provided in Section IV.A. We use Monte Carlo simulation to generate a profit and loss distribution for the buyer of this sample contract. The distribution is shown in Figure 1 below.

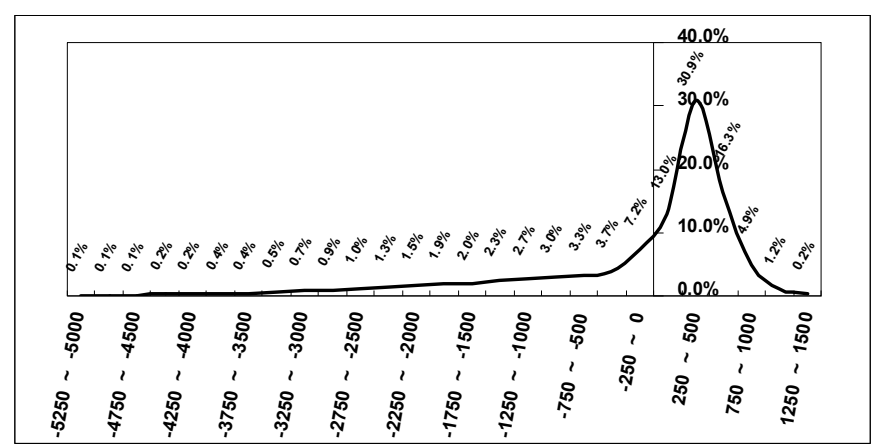

Fig. 1. Probability Distribution Function of Profit and Loss of the sample accumulator

It is clear from Figure 1 that the profit/loss distribution is very asymmetrical. It has a long left tail meaning that extreme loss is possible. On the other hand, extreme profit is unlikely as the distribution has a short right tail. This has an easy interpretation because if the market is bullish, the contract will be knocked-out once the price breaches the barrier $H$. However, when the market is very bearish, the loss can be very substantial. In fact, the total loss can run higher than the notional value of the contract, given the usual definition of notional value. Knowing the profit/loss distribution, one can easily locate its lower 5-percentile. For the sample accumulator contract under our analysis, the lower 5-percentile is $-\$ 2424.50$. This means that at the finish of the contract, there is a $5 \%$ chance to run a loss more than $\$ 2424.50$. For the seller of the contract, we can estimate his/her corresponding loss using the same confidence level 0.95. Computation result shows that the value at risk at contract finish is $\$ 841.01$ with $95 \%$ confidence. Because of the difference between the two values at risk, we can conclude that the seller runs a much smaller risk than the buyer. Notice that the value at risk mentioned here is a little different from the value at risk with a fixed time frame. The value at risk here involves a variable time frame equal to the contract's life, which is random because of the knock-out barrier.

Other than providing a value at risk for the sample accumulator, we can also calculate its Greeks for risk analysis. In particular we can calculate the delta, the gamma and the vega for our sample accumulator. In Appendix III, we provide the formulas for such Greeks. To obtain the Greeks for the sample accumulator with immediate settlement, we simply need to substitute $S_{0}=100, H=105$, $K=90, r=0.03, q=0, \sigma=0.20$ and $n=252$ into the formulae. For delay settlement, the monthly settlement times have to be inputted into the formulae. The numerical values 
for the Greeks are tabulated in Table IV below.

TABLE IV

GREEKS OF ACCUMULATOR CONTRACTS

\begin{tabular}{|c|c|c|c|c|c|c|}
\hline Spot p & ce $S$ & 88 & 92 & 96 & 100 & 104 \\
\hline \multirow{3}{*}{$\begin{array}{l}\text { Immediate } \\
\text { settlement }\end{array}$} & Delta & 290.12 & 211.95 & 137.19 & 65.98 & -3.54 \\
\hline & Gamma & -18.49 & -19.40 & -18.13 & -17.55 & -16.23 \\
\hline & Vega & -12139 & -12507 & -11182 & -8072 & -2966 \\
\hline \multirow{3}{*}{$\begin{array}{c}\text { Delay } \\
\text { settlement }\end{array}$} & Delta & 288.05 & 209.63 & 134.88 & 63.48 & -6.47 \\
\hline & Gamma & -18.67 & -19.35 & -18.16 & -17.62 & -16.37 \\
\hline & Vega & -12201 & -12554 & -11220 & -8100 & -2978 \\
\hline
\end{tabular}

It shows that delta, gamma, and vega are all sizable because an accumulator contract is composed of many option contracts with different expiration days. What is worth noting is that there is an asymmetry in the delta and vega values. When the spot price is low (say $S=88$ ), the magnitude of delta and vega values are much larger than when the spot price is high (say $S=104$ ). Take delta as an example. Delta values are decreasing function of $S$ because gamma values remain at a negative level. Delta has a magnitude of 288.05 (discrete settlement) when $S=88$, but its magnitude drops to -6.47 when $S=104$. This means that losing buyers will be more vulnerable to price changes than winning buyers. Similarly vega has a magnitude of 12201 when $S=88$, but drops to a magnitude of 2978 when $S=$ 104 meaning that compared to winning buyers, losing buyers are more vulnerable to volatility changes as well. This may be one reason why some buyers of the contract become very desperate when the market turns south in recent months. This asymmetry is consistent with the finding that the value at risk of the buyer is several times that of the seller.

\section{CONCLUSIONS}

In this paper, we first derive pricing formulae for an accumulator with immediate settlement. Noting that the difference between immediate settlement and delay settlement. is in whether there is delivery of stocks or delivery of forward contracts on the stock, we are able to generalize the formula to price an accumulator with delay settlement. However, these close form formulae are valid only when the barrier is applied continuously. When the barrier is applied discretely, the formulae need adjustment. Following similar methods in pricing discrete barrier options, we are able to propose approximate formulae to price an accumulator with discrete barrier. Using a numerical example, we show that the approximation is pretty accurate. Based on the analytical formulae on accumulator pricing, we also study its fair value, implied volatility, value at risk and the Greeks. The results show that for a buyer, there exists a great asymmetry in terms of risk when market condition is favorable or unfavorable. Although in this paper we only analyzed the most typical accumulator contract, our methods can be easily generalized to some more sophisticated accumulators with exotic features such as multi-asset underlying, partial barrier with protected period or revivable barrier, cross reference with different accumulation asset and reference asset and so on.

\section{APPENDIX I: DERIVING THE PRICING FORMULA FOR AN ACCUMULATOR WITH IMMEDIATE SETTLEMENT}

According to (2) in the main text, we need to compute $C_{u o}$ and $P_{u o}$. Suppose $X_{t}=\sigma W_{t}+\mu t$ is the random process of the asset's logarithmic risk-neutral return, then barrier options on this asset with strike price $K$ and barrier $H$ $(H>K)$ have values at time 0 like the following representation (see Musiela and Rulkowski (1997))

$$
\begin{aligned}
& C_{\text {uо }}=e^{-r t} S_{0} E_{P}\left(e^{X_{t}} \mathrm{I}_{A}\right)-e^{-r t} K P\{A\} \\
& P_{u o}=e^{-r t} K P\{B\}-e^{-r t} S_{0} E_{P}\left(e^{X_{t}} \mathrm{I}_{B}\right)
\end{aligned}
$$

where $A$ and $B$ stand for the events $A=\left\{\omega \in \Omega \mid X_{t} \geq \ln \left(K / S_{0}\right), M_{t}<\ln \left(H / S_{0}\right)\right\}$,

$B=\left\{\omega \in \Omega \mid X_{t}<\ln \left(K / S_{0}\right), M_{t}<\ln \left(H / S_{0}\right)\right\}$ and $M_{t}=\max _{0 \leq \tau \leq t} X_{\tau}$.

According to Rubinstein and Reiner (1991), the density of the underlying risk-neutral asset return $u$ is

$$
f(u)=\frac{1}{\sigma \sqrt{2 \pi t}} e^{-\frac{(u-\mu t)^{2}}{2 \sigma^{2} t}} \quad \text { where } \mu=r-q-\frac{1}{2} \sigma^{2}
$$

Also, the density of the underlying asset return, given that the asset price first starts at $S$ below the barrier $H$, crosses the barrier at least once, but ends up below than $H$ at expiration:

$$
g(u)=e^{2 \mu \alpha / \sigma^{2}} \frac{1}{\sigma \sqrt{2 \pi t}} e^{-\frac{(u+2 \alpha+\mu t)^{2}}{2 \sigma^{2} t}} \quad \text { where } \alpha=\log \left(\frac{H}{S}\right)
$$

Using these density functions, the expectations in $\left(^{*}\right)$ can be computed. With the help of the following notations:

$$
\begin{aligned}
& x=\frac{\log (S / K)+\left(\mu+\sigma^{2}\right) t}{\sigma \sqrt{t}} x_{1}=\frac{\log (S / H)+\left(\mu+\sigma^{2}\right) t}{\sigma \sqrt{t}} \\
& \mu=r-q-\sigma^{2} / 2 \\
& y=\frac{\log \left(H^{2} / S K\right)+\left(\mu+\sigma^{2}\right) t}{\sigma \sqrt{t}} y_{1}=\frac{\log (H / S)+\left(\mu+\sigma^{2}\right) t}{\sigma \sqrt{t}} \\
& \lambda=1+\frac{\mu}{\sigma^{2}}
\end{aligned}
$$

The terms in $(*)$ can be computed as follows:

$$
\begin{aligned}
& E_{P}\left(e^{X_{I}} \mathbf{I}_{A}\right)=\int_{\log \left(\frac{K}{S}\right)}^{\log \left(\frac{H}{S}\right)} e^{u} f(u) d u-\int_{\log \left(\frac{K}{S}\right)}^{\log \left(\frac{H}{S}\right)} e^{u} g(u) d u \\
& =e^{(r-q) t}\left\{N(x)-N\left(x_{1}\right)+\left(\frac{H}{S}\right)^{2 \lambda}\left[N(-y)-N\left(-y_{1}\right)\right]\right\}
\end{aligned}
$$




$$
\begin{aligned}
& E_{P}\left(e^{X_{i}} \mathbf{I}_{B}\right)=\int_{-\infty}^{\log \left(\frac{K}{S}\right)} e^{u} f(u) d u-\int_{-\infty}^{\log \left(\frac{K}{S}\right)} e^{u} g(u) d u \\
& =e^{(r-q) t}\left\{N(-x)-\left(\frac{H}{S}\right)^{2 \lambda} N(-y)\right\} \\
& P\{A\}=\int_{\log \left(\frac{K}{S}\right)}^{\log \left(\frac{H}{S}\right)} f(u) d u-\int_{\log \left(\frac{K}{S}\right)}^{\log \left(\frac{H}{S}\right)} g(u) d u \\
& =N(x-\sigma \sqrt{t})-N\left(x_{1}-\sigma \sqrt{t}\right) \\
& +\left(\frac{H}{S}\right)^{2 \lambda-2}\left[N(-y+\sigma \sqrt{t})-N\left(-y_{1}+\sigma \sqrt{t}\right)\right] \\
& P\{B\}=\int_{-\infty}^{\log \left(\frac{K}{S}\right)} f(u) d u-\int_{-\infty}^{\log \left(\frac{K}{S}\right)} g(u) d u \\
& =N(-x+\sigma \sqrt{t})-\left(\frac{H}{S}\right)^{2 \lambda-2} N(-y+\sigma \sqrt{t})
\end{aligned}
$$

So barrier call option on underlying asset has value

$$
\begin{aligned}
& C_{u o}=e^{-r t} S_{0} E_{P}\left(e^{X_{t}} \mathrm{I}_{A}\right)-e^{-r t} K P\{A\} \\
& =S_{0} e^{-q t}\left\{N(x)-N\left(x_{1}\right)+\left(\frac{H}{S}\right)^{2 \lambda}\left[N(-y)-N\left(-y_{1}\right)\right]\right\} \\
& -e^{-r t} K\left\{\begin{array}{l}
N(x-\sigma \sqrt{t})-N\left(x_{1}-\sigma \sqrt{t}\right) \\
+\left(\frac{H}{S}\right)^{2 \lambda-2}\left[N(-y+\sigma \sqrt{t})-N\left(-y_{1}+\sigma \sqrt{t}\right)\right]
\end{array}\right.
\end{aligned}
$$

Similarly, barrier put option on underlying asset has value $P_{u o}=e^{-r t} K P\{B\}-e^{-r t} S_{0} E_{P}\left(e^{X_{t}} \mathrm{I}_{B}\right)$

$$
\begin{aligned}
& =e^{-r t} K\left\{N(-x+\sigma \sqrt{t})-\left(\frac{H}{S}\right)^{2 \lambda-2} N(-y+\sigma \sqrt{t})\right\} \\
& -S_{0} e^{-q t}\left\{N(-x)-\left(\frac{H}{S}\right)^{2 \lambda} N(-y)\right\}
\end{aligned}
$$

These results lead to the formula (3) and (4).

\section{APPENDIX II: DERIVING THE PRICING FORMULA FOR AN ACCUMULATOR WITH DELAY SETTLEMENT}

For delay settlement, forward contracts are delivered instead of stocks. In the main text, the pricing formula (6) involves $C_{u o}^{F}$ and $P_{u o}^{F}$. Since the value of a forward contract is related to the spot value, we have

$$
\begin{aligned}
& C_{u o}^{F}=e^{-r T} S_{0} E_{P}\left(e^{X_{t}} I_{A}\right) E_{p}\left(e^{X_{T}-X_{t}}\right)-e^{-r T} K P\{A\} \\
& P_{u o}^{F}=e^{-r T} K P\{B\}-e^{-r T} S_{0} E_{P}\left(e^{X_{t}} I_{B}\right) E_{p}\left(e^{X_{T}-X_{t}}\right)
\end{aligned}
$$

Since the density functions involved are the same as that in Appendix 1, the terms in $(* *)$ are the same as the terms in (*) except a constant factor. Hence, we get the pricing formula below for options on forward contracts:

$$
\begin{aligned}
& C_{u o}^{F}=e^{-r T} S_{0} E_{P}\left(e^{X_{i}} \mathrm{I}_{A}\right) E_{p}\left(e^{X_{T}-X_{t}}\right)-e^{-r T} K P\{A\} \\
& =S_{0} e^{-q T_{j}}\left\{N(x)-N\left(x_{1}\right)+\left(\frac{H}{S}\right)^{2 \lambda}\left[N(-y)-N\left(-y_{1}\right)\right]\right\} \\
& -e^{-r T_{f}} K\left\{\begin{array}{l}
N(x-\sigma \sqrt{t})-N\left(x_{1}-\sigma \sqrt{t}\right) \\
+\left(\frac{H}{S}\right)^{2 \lambda-2}\left[N(-y+\sigma \sqrt{t})-N\left(-y_{1}+\sigma \sqrt{t}\right)\right]
\end{array}\right\} \\
& P_{u o}^{F}=e^{-r T} K P\{B\}-e^{-r T} S_{0} E_{P}\left(e^{X_{t}} \mathbf{I}_{B}\right) E_{p}\left(e^{X_{T}-X_{t}}\right) \\
& =e^{-r T} K\left\{N(-x+\sigma \sqrt{t})-\left(\frac{H}{S}\right)^{2 \lambda-2} N(-y+\sigma \sqrt{t})\right\} \\
& -S_{0} e^{-q T}\left\{N(-x)-\left(\frac{H}{S}\right)^{2 \lambda} N(-y)\right\}
\end{aligned}
$$

\section{APPENDIX III: THE GREEKS}

Formulae for an accumulator's delta, gamma and vega can easily be obtained through differentiation. We provide below those with delay settlement. For the calculation process, please refer to Kerry Back (2005) and it provides the Greeks's calculation details for vanilla option.

$$
\begin{aligned}
& \text { Delta } \\
& \frac{\partial V}{\partial S}
\end{aligned}
$$

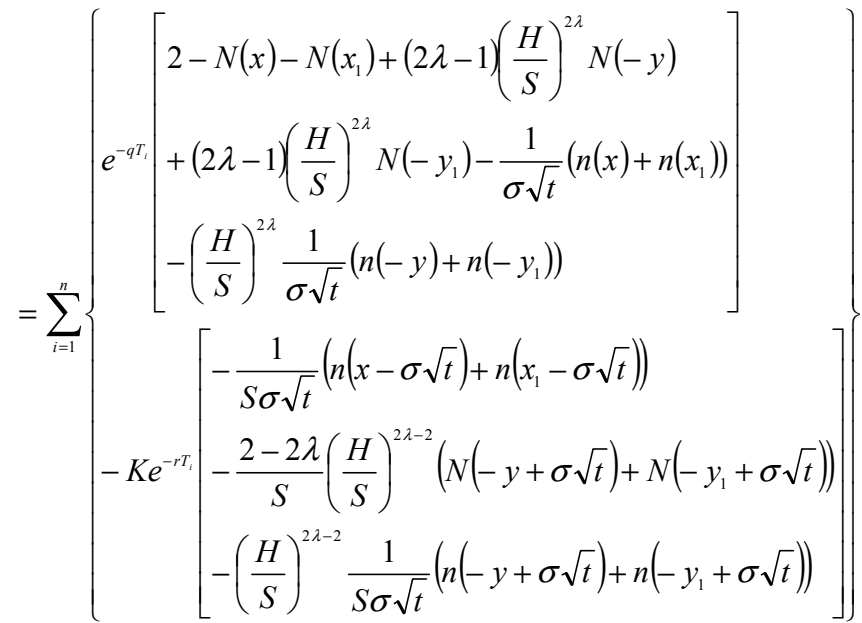

where $x, x_{1}, y, y_{1}$ are short for $x\left(t_{i}\right), x_{1}\left(t_{i}\right), y\left(t_{i}\right)$, $y_{1}\left(t_{i}\right)$ with same functions defined in Appendix I but different values of time. $H$ can be substituted by $\widetilde{H}_{i}=H e^{\beta \sigma \sqrt{T_{i} / m_{i}}}$ to pricing the delta of an accumulator with discrete barrier.

\section{Gamma}




$$
\begin{aligned}
& \frac{\partial^{2} V}{\partial S^{2}}
\end{aligned}
$$

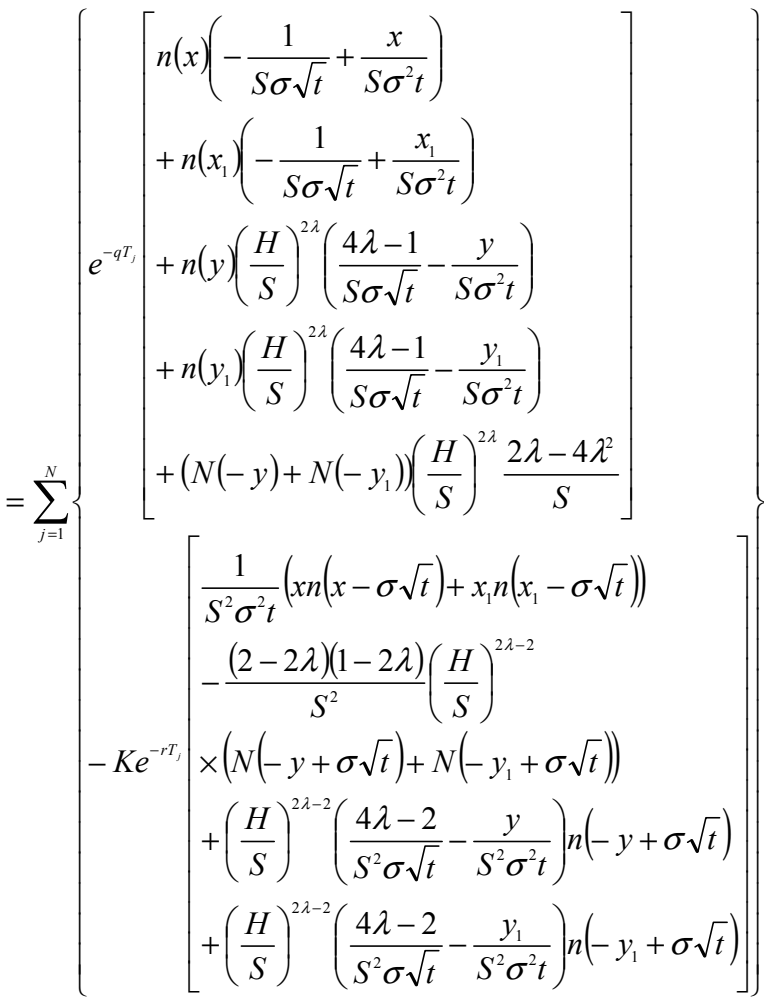

where $H$ can be substituted by $\widetilde{H}_{i}=H e^{\beta \sigma \sqrt{T_{i} / m_{i}}}$ to pricing the gamma of an accumulator with discrete barrier.

$$
\begin{aligned}
& \text { Vega } \\
& \frac{\partial V}{\partial \sigma}
\end{aligned}\left\{\begin{array}{l}
S e^{-q T(j)}\left[\begin{array}{l}
-n(x)\left(\sqrt{t}-\frac{x}{\sigma}\right)-n\left(x_{1}\right)\left(\sqrt{t}-\frac{x_{1}}{\sigma}\right) \\
+\ln \left(\frac{H}{S}\right)\left(\frac{H}{S}\right)^{2 \lambda} \frac{4(r-q)}{\sigma^{3}}\left(N(-y)+N\left(-y_{1}\right)\right) \\
+n(y)\left(\frac{H}{S}\right)^{2 \lambda}\left(\sqrt{t}-\frac{y}{\sigma}\right)+n\left(y_{1}\right)\left(\frac{H}{S}\right)^{2 \lambda}\left(\sqrt{t}-\frac{y_{1}}{\sigma}\right) \\
{\left[\begin{array}{l}
n(x-\sigma \sqrt{t}) \frac{x}{\sigma}+n\left(x_{1}-\sigma \sqrt{t}\right) \frac{x_{1}}{\sigma} \\
+\ln \left(\frac{H}{S}\right)\left(\frac{H}{S}\right)^{2 \lambda-2} \frac{4(r-q)}{\sigma^{3}} \\
\times\left(N(-y+\sigma \sqrt{t})+N\left(-y_{1}+\sigma \sqrt{t}\right)\right) \\
-n\left(-y+\sigma \sqrt{t} \frac{y}{\sigma}\right)\left(\frac{H}{S}\right)^{2 \lambda-2}
\end{array}\right.}
\end{array}\right] \\
-X e^{-r T(j)} \\
-n\left(-y_{1}+\sigma \sqrt{t}\right) \frac{y_{1}}{\sigma}\left(\frac{H}{S}\right)^{2 \lambda-2}
\end{array}\right]
$$

For accumulator with discrete barrier, because the correction term contains volatility parameter, we also need to differentiate it respect to volatility when valuing vega. So the formula is modified to

$$
\begin{aligned}
& \frac{\partial V_{\text {discrete }}}{\partial \sigma}
\end{aligned}
$$

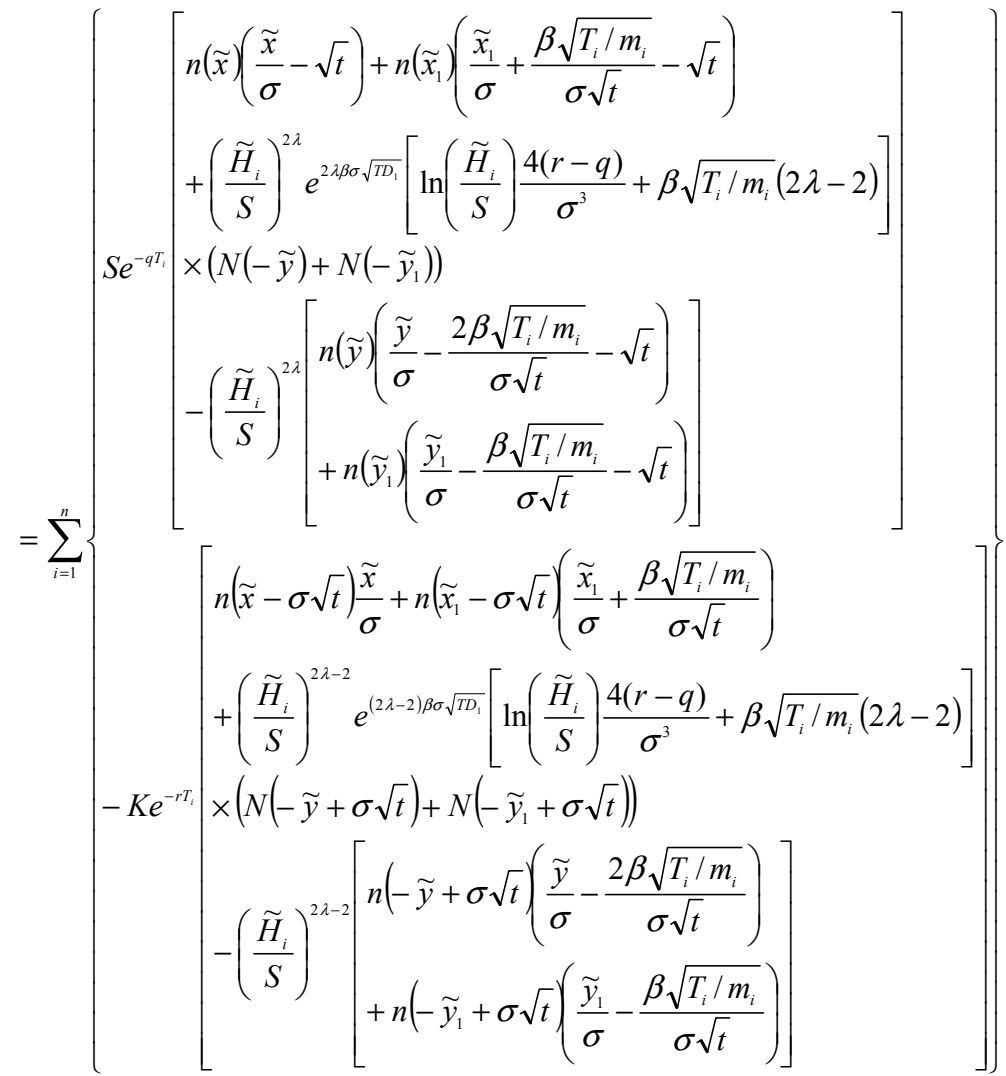

where $\tilde{x}, \tilde{x}_{1}, \tilde{y}, \tilde{y}_{1}$ are short for $x\left(t_{i}, \tilde{H}_{i}\right), x_{1}\left(t_{i}, \tilde{H}_{i}\right)$, $y\left(t_{i}, \tilde{H}_{i}\right), y_{1}\left(t_{i}, \widetilde{H}_{i}\right)$.

\section{REFERENCES}

[1] PP. Boyle and S.H. Lau, "Bumping up against the barrier with the binomial method," Journal of Derivatives, 1994, 1, 6, 4.

[2] M. Broadie, P. Glasserman and S.G. Kou, "A continuity correction for discrete barrier Options," Math. Finance, 1997, 7, 325-348.

[3] M. Broadie, P. Glasserman and S.G. Kou, "Connecting discrete and continuous path-dependent options," Finance Stochastics, 1999, 3, 5582.

[4] T. Cheuk, and T. Vorst, "Complex barrier options," Journal of Derivatives, 1996, 4.8 .

[5] J.M. Harrison, Brownian Motion and Stochastic Flow Systems. New York: Wiley

[6] R. Heynen, and H. Kat, "Partial barrier options," J.Financial Eng. 1994, 3, 353-74.

[7] Kerry Back, A Course in Derivative securities: Introduction to Theory and Computation. 2005, Chaper 3, P53-P55. Springer

[8] Musiela Marek and Rutkowski Marek, Martingale Methods in Financial Modelling. 1997, Springer.

[9] M. Reimer and K. Sandmann, "A Discrete Time Approach for European and American Barrier Options," 1995, working paper.

[10] P. Ritchken, "On pricing barrier options," Journal of Derivatives, 1995, 3, 19-28

[11] Mark Rubinstein and Eric Reiner, "Unscrambling the binary code," Risk, 1991, 4(9), 75-83.

[12] Smann Matthias and A. Klaus, "A Discrete Time Approach for European and American Barrier Options," 1995, Working Paper, Department of Statistics, University of Bonn

[13] Uwe Wystup, FX options and structured products, 2006, Chichester, England: Wiley.

[14] M.J. Schervish, Algorithm AS 195: Multivariate normal probabilities with error bound. Appl. Statist., 1984, 33, 81-94. 